\title{
A COMPANY'S LONG-TERM INNOVATION CAPACITY WHEN INTRODUCING ENTERPRISE SOFTWARE: SOCIOLOGICAL ASSESSMENT OF HYBRID CLOUD-PLATFORMS
}

\author{
Marek Holze \\ University of Duisburg-Essen \\ Universitätsstraße 2, 45141 Essen, Germany
}

\begin{abstract}
While the cloud emerged as an economic successful model compared to Application Service Provider (ASP), it is now a prerequisite for ever shorter innovation cycles in networked companies; risks and opportunities arise for companies using enterprise systems. Startup companies are positioned to implement innovations and build up the company, as well as its further components, while taking innovation into account. Established companies have to deal with the integration of these innovations into their existing business system. This paper studies the aspects that must be considered when software is introduced to bring about a stable innovation capability for the company. A case study is therefore presented in which a medium-sized retail company introduces new POS (point-of-sale) software with the help of a cloud platform. The case study assessed the relevance of various aspects, which must be considered during implementation. The relevance of complexity reduction was, among others, rated as highly relevant while uncertainty absorption, in contrast to other literature, was rated as less relevant.
\end{abstract}

KEYWORDS

Complexity Reduction, Uncertainty Absorption, Platform, Ecosystem, Standard Software, Cloud

\section{INTRODUCTION}

Brown and Vessey (2008) have already indicated that better managing the complexity of enterprise systems reduces the risk of failure and the costs involved. For the second "Wave of Enterprise Systems" a reduction of sociological complexity is therefore strategically important for a company. When it comes to implementing enterprise software, complexity becomes an important issue for academic researchers and practitioners around the world as Hwang (2005) stressed. In Europe it stressed Hanseth et. al. (2001); in Asia it stressed Liang et. al. (2004) and Martinsons (2004). If the core performance's complexity increases, for example by integrating new software products into an existing IT landscape, complexity must be reduced elsewhere. If this is not considered, new resources are required in the first step, for example for managing new IT systems. This paper must realize that not only the time expenditure, but also the necessary understanding of the associated components increases in order to forecast the long-term consequences of changes - in summary, qualitative and quantitative expenditure increases. In the following, the qualitative effort is referred to as complexity. While the quantitative effort can be dealt with almost without limitations - with sufficient capital -, the manageable complexity is limited by the intelligence of the employees or consultants. This complexity can hardly be dealt with by using a larger number of IT staff; this is often even counterproductive. When integrated innovations are introduced into the enterprise system, another part of the system should simultaneously be relieved of complexity. Cloud platforms can possibly reduce the enterprise system's complexity. As a rule, no new IT system is created for an organization - a digital core ERP is used onsite, which is supplemented by functionalities via a cloud platform. The leaner the core ERP (enterprise resource planning) is, the less complexity has to be handled in the company - this is widely known as standard software implementation. Furthermore, uncertainty absorption is mostly promoted by integrated and software-based introductions. In the latter, decisions are linked to previous decisions. 
This encourages workflow efficiency and effectiveness in the company, as Hwang (2005) stressed. This paper studies the following research question via a case study:

$$
\begin{aligned}
& \text { How can a hybrid cloud-platform approach promote the long-term innovation capability of enterprise } \\
& \text { systems? } \\
& \text { What aspects must be considered in such an introduction? }
\end{aligned}
$$

When complexity is reduced in companies using enterprise systems, they remain innovative with less effort, which prevents displacement by competitors. In other words: Companies that use enterprise systems perceive the network effects, which software manufacturers are striving for, as pressure to innovate. These companies will thus become more digital and less technical - a prerequisite for the ever-shorter innovation cycles. This paper uses a case study to research the introduction of new standard software to replace individual software. A hybrid cloud-platform approach is used for introducing new software.

In order to answer the research question, the second chapter introduces basic technological terms, for further study, based on a historical overview of ERP systems. The third chapter explains the structure of the case study and presents the results. This is followed, in the fourth chapter, by a discussion of the case study's results. This paper concludes with a summary of the research results.

\section{HISTORY OF ERP AND ONGOING CHANGES}

The history of ERP (enterprise resource planning) systems is significant for understanding enterprise systems, their ability to innovate, and some of the problems associated with them. For this reason, this paper presents a broad historical review in the following. In the 1960s, when computers were first used in business operations, application systems were developed for production planning and control purposes, as discussed by Görtz and Hesseler (2007). Individual companies usually developed these application systems into an individual software package to meet specific requirements (Kurbel 2016). However, with ever increasing hardware performance, the application scope of the systems expanded, which was also expressed by the name Manufacturing Resource Planning Systems (MRP II) (Schütte \& Vering 2011). The term Enterprise Resource Planning (ERP) was coined by the Gartner Group (Jung-Elsen 2013).

The Application Service Provider (ASP) concept, which is known since 1997, represents a further development of IT outsourcing in the direction of today's SaaS. Hess and Wolf (2008) cite customer-specific infrastructure as the main reason for ASP's lack of profitability. Hess et. al. (2009) looks into this problem by means of a study and deals with the change from ASP to SaaS or ERPaaS (enterprise resource planning as a service). This study determines the demand for SaaS (software as a service), especially for users with low complexity and criticality.

Gottwald (2013) highlights the aspiration of companies to anchor ERP solutions deeply in their own corporate structure and to use them for a correspondingly long time. From the user's point of view, the focus is not on being up-to-date, but rather on purpose and function. In order to represent these aspects in the ERP system, they are usually highly individualized. Elsewhere, a less holistic stance is taken towards onsite ERP systems: While Josefiok (2014) concludes that it seems unlikely to realize a holistic solution with the current offer of ERPaaS and the SaaS providers' orientation towards less individualized products, Jung-Elsen (2013) shows that ERPaaS is mostly used as an addition to existing ERP systems -these additions are usually included in areas such as CRM (customer relationship management), collaboration, and HR (human resource management). There, the advantages of a cloud - such as location-independent access - would be particularly noticeable. This paper confirms that this statement is true for retailers. Furthermore, it shows that very few providers offer traditional ERP via a public cloud service. In most cases it concerns hosting company-specific needs in the cloud infrastructure. 


\section{CASE STUDY}

A qualitative case study presents the results of interviews with a medium-sized retail company during a five-month software introduction in the summer of 2018. The study was carried out by the author of this article and is organized as Oates (2005) described. The following two chapters present the case study and examine the results of the interviews.

\subsection{The Case Study}

The company chosen for the case study, has approximately 300 stores in Germany. In 2013, the company had a turnover of 130 million euros. The current ERP system is hosted in a cloud. This is not ERPaaS, because the software as such is completely and separately available. A hybrid cloud-platform approach was chosen for introducing the new POS software. The hosted version is also selected for the standard POS software. When the software is introduced, the branch stores will be equipped with new cash registers. All functions, which a store must perform, such as cashing up daily or receiving new goods, are newly implemented. This results in process and ERP system changes. Two new software solutions are introduced in the stores: The POS software itself and a so-called "portal" on a separate computer in which functionalities are provided independently of the actual sales process. Both the new POS software and the ERP system are standard software. The previous POS software was developed by the retail company itself. Three parties are involved in the implementation of the project: The retail company itself, a consultant of the POS software manufacturer, and an independent consulting company. The independent consulting firm also advised on the ERP implementation. Eighteen interviews were conducted with all three parties for the case studies. The interview partners are broken down into the project roles as shown in Table 1.

Table 1. Case Study Setup

\begin{tabular}{l|cccc} 
& Project Leader & Consultan & $\begin{array}{c}\text { Specialist } \\
\text { Department }\end{array}$ & IT Department \\
\hline Retail company & 1 & 2 & 3 & 3 \\
POS software manufacturer & 1 & 2 & - & - \\
Consultancy firm & 1 & 2 & - & 3
\end{tabular}

Unstructured interviews were conducted so that, over a period of five months, a direction of questioning could be determined without, as far as possible, leaving out any facets of the software introduction and without determining the focus in advance. For the case study, concepts for introducing the new software, as well as concepts of the ERP project and the old POS software were analyzed. During the software introduction, processes were identified, which could not be handled in the standard POS software. The ERP manufacturer's app platform was used to develop the lacking functionalities. For example, certain reports could be used; certain apps had to be re-developed by using the tools, which were made available in the cloud platform to create mockups and programming. The classic consulting concept was used here, in which consultants work together with the specialist department to develop a concept for the apps. The retail company's IT department then collaborates with the technical consultants of the independent consulting company to implement these, which are not available on the platform.

\subsection{Case Study Results}

Next, this paper presents the interview results. Figure 1 shows the topics resulting from the document analysis on the y-axis, subdivided according to the survey groups. The $\mathrm{x}$-axis shows the number of interview partners who consider the respective topic as important. It becomes clear that complexity reduction plays an important role when it comes to introducing the new standard software. The topic in the interviews related to a "close-to-standard software introduction." This means that as few new developments as possible were made so that the maintenance complexity was reduced and innovations or new releases could be implemented more quickly. This topic is indirectly linked to lower implementation costs. The topic of uncertainty absorption is given less priority when it comes to introducing POS software. The interviews clearly showed that this characteristic for introducing the ERP system is rated almost on the same level as complexity reduction. The 
reason for this is that uncertainty absorption is primarily driven by ERP systems. With the new POS software, this topic plays a relatively minor role. The other topics were evaluated in the interviews, but with less relevance for the software introduction project and are not considered further in this paper.

While Sontow and Kleinert (2010) emphasize the relatively high dependence on service providers and the very consistent standardization of the software, the interviews in this case study show otherwise. Becker and Ortmann (2010) already pointed out that ERP systems are in use for more than 10 years on average in small and medium-sized enterprises, as well as in large enterprises. Under this premise, the hosted version of an ERP system was chosen. This, of course, makes it less likely that the service performance will be rated as good overall, since only one of the two service providers - namely the software manufacturer or the hosting service provider - needs to work unsatisfactorily. In the interviews, the question about the fear of lock-in effects was answered by the fact that it would be very difficult for a medium-sized company to provide comparable services in a short time as a cloud operator would do. This includes technical aspects, as well as personnel. In addition, an internally-hosted system could also be described as a lock-in effect, since employees need to be trained. Switching to another system would hardly be less complicated.

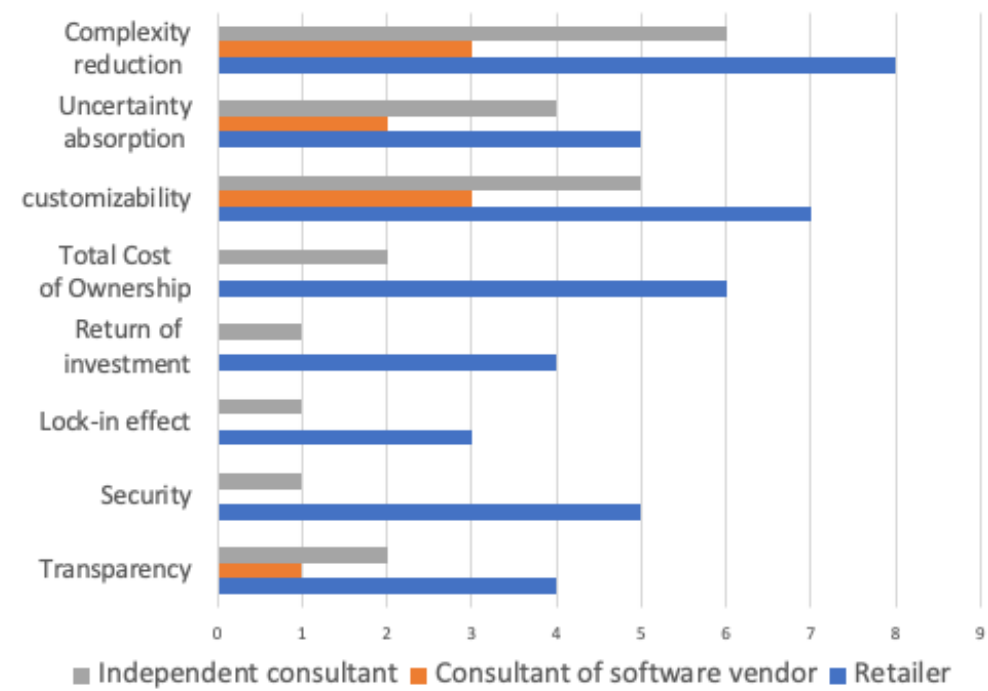

Figure 1. Rating of Importance of Factors for Hybrid Cloud- platform Software Implementation

\section{EFFECTS OF A HYBRID CLOUD-PLATFORM ARCHITECTURE}

Next, this paper discusses the decision, which was made in the introductory project, to adopt a hybrid cloud platform, taking into account various aspects; these are shown in Figure 2. Complexity reduction and uncertainty absorption are investigated analogously to the results of the interviews. While preparating for the following discussion, the sociological complexity theory - in the context of a cloud platform -will be inferred; this gives rise - almost incidentally - to a sociological legitimation of SaaS providers with which the retail company's decision can be placed in a scientific context. 


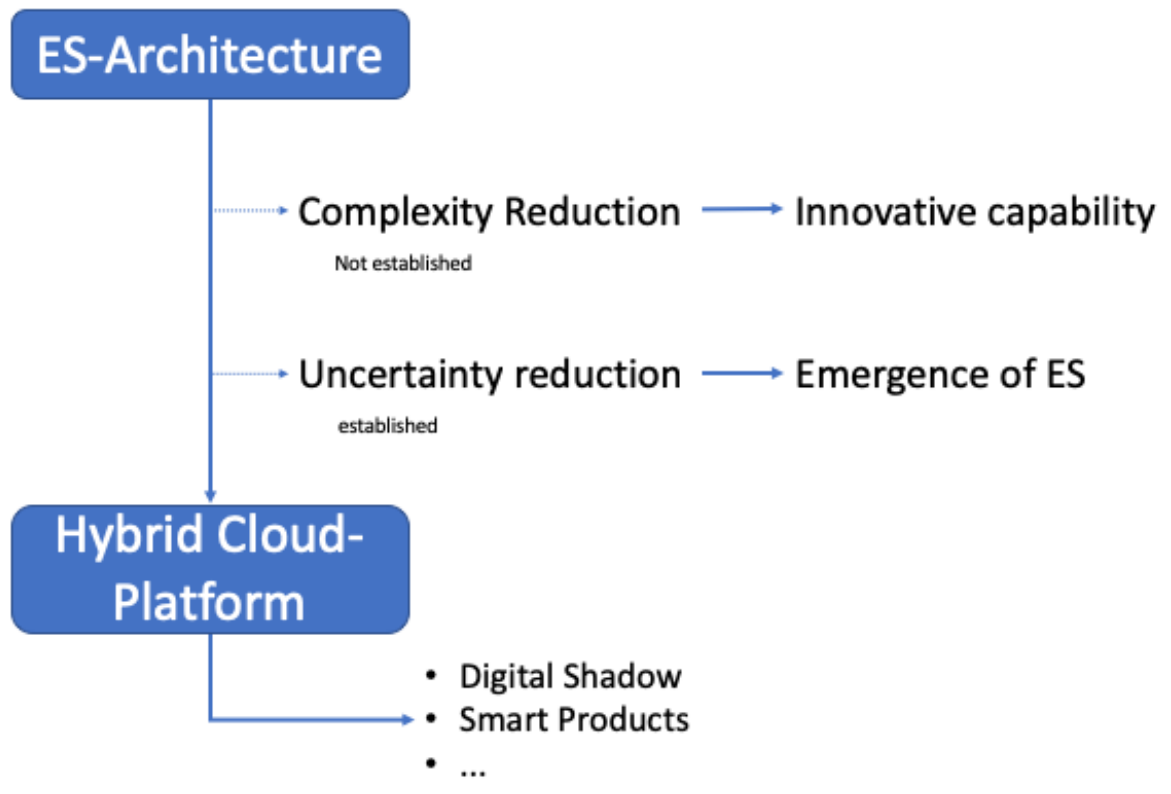

Figure 2. On-going changes of ES-Architecture

\subsection{Social Legitimacy of SaaS Providers}

In general, a system is a dynamic entity with certain characteristics and behaviors (Ropohl 2009). It consists of interconnected parts. No part is independent of all the other parts and the behavior of the unit is influenced by the interaction of all parts. Subsystems must fulfill two tasks for a form of system differentiation: A subsystem recognizes the essence of another subsystem, and the subsystem is defined according to this difference (Luhmann 1997). Subsystems differ in that they diagnose a social problem and use their processing and solution functionalities. Companies are subsystems related to this work. As an enterprise, the subsystem tries to legitimize society as a whole in order to differentiate itself further; the subsystem does this by adopting a certain function and by providing a service.

The modern economy often contains a differentiation, which is brought about by the increasing complexity of individual tasks and areas. In this paper, Software-as-a-Service is considered as a consequence of the technological world's increasing complexity. SaaS providers solve the high-complexity problem of software and its necessary infrastructure. This socially legitimizes SaaS providers. The previous chapter already stated that, according to Hess et. al. (2009), the ASP model was not ecologically successful, due to customer-specific infrastructure. The complexity, which was previously created at individual companies in the form of infrastructure, was identically reproduced in the ASP. This does not lead to any reduction in complexity and can therefore only achieve ecological advantages with difficulty. Only when a cloud is introduced can complexity be reduced, thereby legitimizing the CSP (cloud solution provider).

\subsection{Complexity Reduction}

The previous chapter discusses the legitimacy of SaaS providers as a process of social differentiation. The complexity argument is used; this is discussed in more detail below. Two possible ways of reducing complexity are investigated.

Operational closure means that the system's behavior affects itself and becomes the starting point for further behavior. Operational consistency in small systems reduces the complexity of each individual system. This can explain why a decentralized IT landscape is often created - almost automatically -in companies. Designing an integrated application system for the entire enterprise is usually a much more complex task. The trend is to outsource such ventures to an external software manufacturer in the form of standard 
software. In accordance with the previous chapter's argument, software vendors can - in addition to SaaS providers -also be socially legitimized in this way.

Second, in their environment, communication systems only perceive what is appropriate to their subject; what is associated with the "Sinn" (meaning) of earlier communication. For Luhmann, meaning is a mechanism for reducing complexity. In the infinitely complex environment, only a small part is filtered out according to certain criteria; the boundary of a social system thus marks a difference in complexity from the outside to the inside. The legitimization of software manufacturers is thus not only determined by their ecological use of network effects -that is, they supply many companies with the same or similar software. According to Luhmann, the complexity, and thus the controllability, of a task decreases, also when systems are distinguished from each other according to meaning. This cannot happen within a company, because the entire company usually has a single meaning - for example, to sell food inexpensively. Overhead cost calculations represent an attempt to break down this meaning within a company. Nevertheless, it remains difficult to link the success or failure of a company to the IT department.

Based on interviews, Johansson and Ruivo (2013) have already reported a decline in the complexity of implementing an ERP in the cloud. According to the interviews of this work, the reduction of complexity played an important role both in the introduction of the new POS software and in the previous ERP projects. The interviews drew attention to the following points on choosing a strategy for introducing the software when it comes to complexity: In the long run, ERP systems will have to be modified time and again. This emerged from the theory of Orlikowski (1992), in which human agents change or influence technology. This is a critical point when it comes to complexity, because resources must be available at all times to anticipate how software changes will impact on the complete enterprise system. As the systems interact with each other in a more integrated way, so the complexity, which needs to be managed, increases. Many software projects still fail due to this - even years after the software introduction. The availability of resources must be taken into account when introducing software. For example, employees can leave the company or a new consultancy company can be appointed. In most cases, the long-term availability of knowledge should be ensured through concepts. Analogous to the point of changes, effects must be understood if a subsequent innovation is to be integrated.

\subsection{Uncertainty Absorption}

Luhmann adopted the term uncertainty absorption from March and Simon (1958). According to Luhmann (2000) and Simon (2007), uncertainty arises when knowledge and lack of knowledge occur simultaneously. It, therefore, arises due to this balance between knowledge and lack of knowledge. Uncertainty absorption occurs when a decision is made after evidence had been gathered, and the conclusions - instead of the evidence- are communicated. This is exactly what usually occurs when an enterprise system is introduced; where decisions are supported by, for example, an ERP system with the help of data or data evaluations. For example, in the following query of a marketing manager to an ERP system, there is no identity between data entry and query: Which advertising campaigns help reduce underutilization in production capacities? The answer to this question consists of marketing data and production data; the sales figures of the product in individual sales areas, as well as the corresponding advertising campaign of the last months are shown to the marketing manager on the basis of this data linking. This means that there is no longer any identity between data input and information retrieval (Mormann 2016). By linking decisions like this, Luhmann succeeds in explaining-from an organizational theory perspective-how the organization establishes itself as a developing order and social system (Luhmann 2000). In the present case study, uncertainty absorption was assessed as rather insignificant in the new software implementation. This may be due to the fact that uncertainty absorption is mainly relevant for decision makers and therefore less important when it comes to introducing a new POS software. Figure 2, therefore, deals with uncertainty absorption.

\section{CONCLUSION AND FUTURE WORK}

This paper presents a case study in which the introduction of a hybrid cloud platform was examined. The results of this case study showed a clear trend towards the importance of complexity reduction. The topic related to a "close-to-standard software introduction." Uncertainty absorption was assigned a lesser 
importance in the new POS software introduction. This is attributed to the ERP system, which was already implemented. Thus, uncertainty absorption seems to depend on the contextual circumstances of a software introduction, and complexity reduction seems to be significant across the board-at least for ERP and POS software introductions. Subsequently, this paper provides a sociological justification for the legitimacy of SaaS. Possible approaches, which could be used to reduce complexity, were explained. In the case study, the relevant aspects for reducing complexity were identified via interviews. Uncertainty absorption was assessed as less relevant for the implementation project. This paper has established how uncertainty absorption relates to the cloud platform topic.

Overall, the issue of complexity reduction continues to be regarded as relevant for both research and practice. Because the relevance of reduction absorption has been identified as dependent on the particular introductory context, this paper expects that interest for research and practice will decline.

\section{REFERENCES}

Becker, M., \& Ortmann, J. (2010). ERP-Studie. Hamburg: mondula GmbH.

Brodocz, A. (1997). Niklas Luhmann: Die Gesellschaft der Gesellschaft. MEDIENwissenschaft: Rezensionen| Reviews, (4), 441-441.

Brown, C. V., \& Vessey, I. (2008). Managing the Next Wave of Enterprise Systems: Leveraging Lessons from ERP. MIS Quarterly Executive, 2(1), 6.

Ciborra, C., Hanseth, O., \& Braa, K. (2001). The control devolution: ERP and the side effects of globalisation. Database for Advances in Information Systems, 32(4).

Gottwald, M. (2013). ERP Trend Report 2013 Prozessindustrie. München: Infor Deutschland GmbH.

Görtz, M., \& Hesseler, M. (2007). Basiswissen ERP-Systeme: Auswahl. Einführung \& Einsatz betriebswirtschaftlicher Standardsoftware, W3I.

Hwang, Y. (2005). Investigating enterprise systems adoption: uncertainty avoidance, intrinsic motivation, and the technology acceptance model. European journal of information systems, 14(2), 150-161.

Hess, T., \& Wolf, C. M. (2008). Software as a service 1.0 and beyond. Software as a Service: Strategische Perspektiven und praktische Bedeutung, München, 8-14.

Hess, T., Benlian, A., Wolf, C. M., \& Buxmann, P. (2009). ERP-as-a-Service: Zukunft oder Sackgasse?. Controlling \& Management, 53(3), 14-17.

Johansson, B., \& Ruivo, P. (2013). Exploring factors for adopting ERP as SaaS. Procedia Technology, 9, 94-99.

Josefiok, M., Göring, M., \& Rohde, J. (2014). ERP as a Service Potenziale und Hemmnisse. ERP Management, 10.

Jung-Elsen, S. (2013). Sind ERP-Systeme geeignet für die Cloud?. In ERP Management, 9.

Kurbel, K. (2016). Enterprise Resource Planning und Supply Chain Management in der Industrie: von MRP bis Industrie 4.0. Walter de Gruyter GmbH \& Co KG.

Liang, H., Xue, Y., Boulton, W. R., \& Byrd, T. A. (2004). Why Western vendors don't dominate China's ERP market. Communications of the ACM, 47(7), 69-72.

Luhmann, N. (2000). Die Paradoxie des Entscheidens. In Organisation und Entscheidung (pp. 123-151). VS Verlag für Sozialwissenschaften.

Luhmann, N. (2000). Vertrauen: Ein mechanismus der reduktion sozialer komplexität (Vol. 2185). Grove/Atlantic, Inc.

March, J. G., \& Simon, H. A. (1958). Organizations.

Martinsons, M. G. (2004). ERP in China: one package, two profiles. Communications of the ACM, 47(7), 65-68.

Mormann, H. (2016). Das Projekt SAP: Zur Organisationssoziologie betriebswirtschaftlicher Standardsoftware. transcript Verlag.

Oates, B. J. (2005). Researching information systems and computing. Sage.

Orlikowski, W. J. (1992). The duality of technology: Rethinking the concept of technology in organizations. Organization science, 3(3), 398-427.

Ropohl, G. (2009). Allgemeine technologie: Eine systemtheorie der technik (p. 363). KIT Scientific Publishing.

Schütte, R., \& Vering, O. (2011). Erfolgreiche Geschäftsprozesse durch moderne Warenwirtschaftssysteme: Produktübersicht marktführender Systeme und Auswahlprozess. Springer-Verlag.

Simon, F. B. (2007). Einführung in die systemische Organisationstheorie (Vol. 1). Heidelberg: Carl-Auer.

Sontow, K., \& Kleinert, A. (2010). Software as a Service-Die schlanke Zukunft für ERP-Lösungen? Ergebnisse eine Anwenderbefragung. ERP Management, 6(4), 24-27. 